\title{
Desenvolvimento de indicadores para avaliação de serviço público de odontologia
}

\author{
Development of indicators \\ for evaluating public dental healthcare services
}

Vera Lucia Ribeiro de Carvalho Bueno ${ }^{1}$

Luiz Cordoni Júnior ${ }^{1}$

Arthur Eumann Mesas ${ }^{2}$

${ }^{1}$ Universidade Estadual de Londrina Campus

Universitário. Rodovia Celso Garcia Cid | Pr 445 Km 380. 86055-900 Londrina PR. veracarvalho11@hotmail.com

${ }^{2}$ Facultad de Medicina, Universidad Autonoma de Madrid.

\begin{abstract}
The objective of this article is to describe and analyze the development of indicators used to identify strengths and deficiencies in public dental healthcare services in the municipality of Cambé, Paraná. The methodology employed was a historical-organizational case study. A theoretical model of the service was developed for evaluation planning. To achieve this, information was collected from triangulation of methods (interviews, document analysis and observation). A matrix was then developed which presents analysis dimensions, criteria, indicators, punctuation, parameters and sources of information. Three workshops were staged during the process with local service professionals in order to verify whether both the logical model and the matrix represented the service adequately. The period for collecting data was from November 2006 through July, 2007. As a result, a flowchart of the organization of the public dental health service and a matrix with two-dimensional analysis, twelve criteria and twenty-four indicators, was developed. The development of indicators favoring the participation of people involved with the practice has enabled more comprehensive and realistic evaluation planning.

Key words Public Health Dentistry, Health Service Evaluation, Healthcare Program and Project Evaluation, Quality Indicators in Healthcare, Process and Outcome Assessment
\end{abstract}

Resumo O objetivo do presente artigo é descrever $e$ analisar o desenvolvimento dos indicadores utilizados para se identificar fortalezas e deficiências de serviço público de odontologia no município de Cambé, Paraná. A metodologia usada foi o estudo de caso histórico-organizacional. Para o planejamento da avaliação foi desenvolvido o modelo-lógico do serviço. Para tanto, foram coletadas informações a partir da triangulação de métodos (entrevistas, análise documental e observação). $\mathrm{Na}$ sequência, foi desenvolvida uma matriz que apresenta dimensões de análise, critérios, indicadores, pontuações, parâmetros e fontes de informações. Três oficinas de trabalho foram realizadas com profissionais do serviço local visando melhor adequação do modelo-lógico e da matriz à realidade do serviço. O período de coleta de dados foi novembro de 2006 a julho de 2007. Como resultado, obteve-se um fluxograma da organização do serviço de odontologia e uma matriz com duas dimensões de análise, doze critérios e vinte e quatro indicadores. O desenvolvimento dos indicadores, privilegiando a participação dos sujeitos envolvidos com a prática, proporcionou o planejamento de uma avaliação abrangente e realista. Palavras-chave Odontologia em Saúde Pública, Avaliação de Serviços de Saúde, Avaliação de Programas e Projetos de Saúde, Indicadores de Qualidade em Assistência à Saúde 


\section{Introdução}

A efetivação dos princípios do Sistema Único de Saúde (SUS) depende de um constante processo de avaliação subordinado aos objetivos e metas do sistema ${ }^{1}$ e, entre outros parâmetros, na análise de indicadores adequados à realidade específica de cada situação ${ }^{2}$.

A incorporação da avaliação dos serviços de saúde é um desafio para a gerência de um sistema complexo como o SUS ${ }^{1}$ e vem sendo debatida pela sociedade brasileira ${ }^{3-5}$. Segundo Felisberto ${ }^{5}$ os avanços no campo da avaliação em saúde, na maior parte dos casos, constituíram-se em iniciativas pontuais que não produziram orientação para a gestão.

Com isto, pesquisadores sugerem a implantação de sistemas de avaliação mais voltados para a captura de dados que reflitam a vivência dos profissionais inseridos no processo de reorientação das práticas de atenção em saúde, incluindo a atenção odontológica $^{6}$. Medina et al. $^{7}$ destacaram que a participação dos grupos envolvidos em um programa é fundamental na elaboração do planejamento da avaliação. Neste sentido, é recomendável que os indicadores a serem analisados estejam fundamentados na necessidade e utilidade específica de cada coletivo, para que os resultados da avaliação possam ser traduzidos em benefícios reais.

Assim, o objetivo deste artigo é descrever e analisar a metodologia utilizada no desenvolvimento de indicadores para avaliação de serviço público de odontologia de Cambé (PR), sendo que a metodologia apresentada privilegia a participação dos profissionais que atuam no serviço de saúde do município.

\section{Metodologia}

\section{Estratégia da pesquisa}

Foi realizado um estudo de caso históricoorganizacional no serviço público de odontologia do município de Cambé (PR). O estudo apresentou três etapas: 1) Elaboração do modelológico da organização do serviço público de odontologia; 2) Elaboração de uma matriz com dimensões de análise, critérios, indicadores, pontuações e parâmetros para avaliação do serviço e 3) Aplicação dos indicadores em uma Unidade Saúde da Família (USF). No presente artigo foram relatadas as duas primeiras etapas.

Segundo Yin $^{8}$, esta modalidade de pesquisa é caracterizada pelo estudo profundo e exaustivo de um ou poucos objetos. No estudo de caso único, do tipo crítico, o pesquisador confronta um modelo-teórico com uma realidade empírica, sendo que este modo de estudo oferece conclusões que poderão eventualmente servir para explicar situações análogas?.

A elaboração do modelo-teórico (modelológico) envolve explicitar os componentes de um programa e suas inter-relações, bem como os fatores relevantes do contexto onde se desenvolve, descrevendo suas potenciais influências sobre os resultados esperados ${ }^{7}$.

Neste estudo, o modelo-lógico foi selecionado como metodologia de trabalho, pois permite apresentar em um esquema visual o modo de funcionamento de um programa e quais resultados podem ser esperados ${ }^{7}$. Este permitiu um estudo detalhado da estrutura organizacional do serviço avaliado.

A apresentação e a discussão deste modelo por gerentes e demais atores implicados, com a utilização tanto de saberes científicos como práticos, permitiu conhecer o objeto da avaliação e definir o que deveria ser medido ${ }^{7}$, sendo utilizado como subsídio para a construção da matriz de indicadores.

A coleta de dados foi realizada entre os meses de novembro de 2006 e julho de 2007.

\section{Local de estudo}

O município de Cambé, localizado ao norte do Paraná, possui uma área total de $495 \mathrm{~km} 2 \mathrm{e}$ apresenta população de 92.888 habitantes ${ }^{10}$, com taxa de mortalidade infantil (12,3 por mil $)^{11}$ inferior à taxa do Estado do Paraná (14,8 por mil $)^{12}$, e indicador de nível educativo, índice de analfabetismo $(10,3 \%)^{11}$ muito próximo $(9,5 \%)^{13}$ ao do Estado do Paraná.

Possui uma rede de serviços públicos de saúde composta por dois hospitais, 12 unidades de Saúde da Família (uma delas com atendimento 24 horas), nas quais atuam 24 equipes de Saúde da Família, sendo 20 com Equipes de Saúde Bucal (ESB) e um Centro de Especialidades Odontológicas (CEO).

O serviço de odontologia começou a se organizar em 1983, a partir da implantação das Ações Integradas de Saúde (AIS), por meio do programa incremental ${ }^{14}$. Posteriormente, houve a inclusão gradativa de pré-escolares e bebês, sendo esta a primeira experiência de Clínica do Bebê na rede pública ${ }^{15}$. O programa Infanto-juvenil contemplava crianças a partir de 24 meses com previsão de acompanhamento até 21 anos $^{14}$. 
Em um contexto que inclui outras medidas coletivas, como a fluoretação da água de abastecimento, em 1987, ${ }^{14}$ é de se destacar que o município obteve conquista significativa na área de saúde bucal, exemplificada pela redução do índice de dentes cariados, perdidos e obturados (CPO-D) de oito dentes em 1983 para menos de um em 2004, para a idade de 12 anos $^{16}$. Porém, uma importante parcela da população adulta tinha acesso somente ao serviço de emergência oferecido em duas unidades de saúde. Neste contexto, surgiram nas conferências municipais de saúde reivindicações para a ampliação do serviç̧o ${ }^{14}$.

Esta ampliação veio ocorrer em setembro de 2005, quando dezoito equipes de Saúde Bucal foram incluídas no PSF, com a finalidade de se reorientar as práticas em saúde bucal com base na Portaria n. $1444^{17}$ do MS e nas Diretrizes da Política Nacional de Saúde Bucal ${ }^{18}$. No ano de 2008 foram incluídas mais duas equipes.

Em 2006, o município recebeu o terceiro lugar na primeira edição do "Prêmio Brasil Sorridente/Conselhos de Odontologia”, uma parceria entre o Conselho Federal de Odontologia (CFO) e o $\mathrm{MS}^{19}$, o que evidencia a importância do serviço público odontológico de Cambé no cenário nacional.

\section{Elaboração do modelo-lógico}

No método adotado, o desenho do modelológico do programa constituiu-se na primeira etapa para o planejamento da avaliação, sendo que a compreensão da organização do serviço em seus diversos níveis de atenção foi fundamental para o desenvolvimento dos indicadores de avaliação.

Para sua construção, o primeiro passo foi coletar informações sobre o programa a partir da triangulação de métodos (observação, análise documental e entrevistas) $)^{7,8}$.

O serviço foi visitado durante duas semanas para a observação das atividades realizadas registrando-se as informações em um caderno de campo. Como fontes de dados secundários foram utilizados o protocolo de atenção odontológica do município ${ }^{20}$ e as Diretrizes da Política Nacional de Saúde Bucal ${ }^{18}$. Para as entrevistas, a pesquisadora contou com a participação de cinco informantes-chaves (o coordenador do PSF, o coordenador da odontologia do serviço de odontologia e três dentistas).

Com base nas informações obtidas, foi possível definir o objeto da avaliação, retratar o seu contexto e identificar elementos da organização do serviço. O esboço do que seria o modelo-lógico foi adaptado do modelo proposto por Vieira-da-Silva et al. ${ }^{21}$, que corresponde à definição de uma imagem-objetivo da descentralização da atenção à saúde em municípios do Brasil, uma vez que, ao se analisar o serviço de odontologia do município ${ }^{20}$, observou-se que o programa está sendo desenvolvido no intuito de reorientar as práticas em saúde bucal com a finalidade de se obter os mesmos resultados propostos pelos referidos autores.

Após esta etapa, foi realizada uma primeira oficina de trabalho (Oficina 1) com a finalidade de se avaliar, junto aos atores envolvidos, se o modelo elaborado representava a lógica do programa. Segundo Anastasiou e Alves ${ }^{22}$, oficina de trabalho pode ser descrita como uma reunião de um pequeno número de pessoas com interesses comuns, a fim de estudar e trabalhar visando ao conhecimento ou aprofundamento de um tema, sob a orientação de um especialista.

A oficina mencionada contou com oito participantes: dois coordenadores do serviço, sendo um do PSF e outro da odontologia, três dentistas que atuam em diferentes unidades Saúde da Família, um mestrando em saúde coletiva, um docente de odontologia da Universidade Estadual de Londrina (UEL) e a pesquisadora principal deste estudo. O convite aos participantes obedeceu pelo menos a um dos seguintes critérios: a) participação desde o início da implantação do atendimento público odontológico no ano de 1983; b) experiência na área de avaliação; c) atuação no serviço público do município; e d) ter sido identificado como informante-chave na primeira fase da pesquisa. Após a adequação do modelo-lógico, foram propostos pelos participantes dessa oficina os aspectos a serem avaliados e que constituiriam a matriz da presente avaliação.

\section{Elaboração da matriz}

Com base nos aspectos a serem avaliados, foi desenvolvida uma matriz que apresenta as dimensões de análise, os critérios, os indicadores, as pontuações e os parâmetros que seriam utilizados para avaliação, bem como as respectivas fontes de informações a serem consideradas. Os seguintes critérios de seleção de indicadores foram utilizados: simplicidade, validade, disponibilidade, robustez, sinteticidade, discriminatoriedade e cobertura ${ }^{7}$.

O primeiro passo para sua construção foi uma revisão de literatura e a elaboração de um esboço de matriz com a seleção de possíveis indi- 
cadores. Realizou-se então uma segunda oficina (Oficina 2), com os mesmos participantes da anterior, com o objetivo de definir a permanência, inclusão ou exclusão de dimensões e indicadores desta matriz de avaliação.

Para a pontuação da matriz foram consultadas diversas fontes de informações: relatórios de produção, protocolo de atendimento ${ }^{20}$, sistemas de informações, revisão de literatura, entrevistas e diário de campo, sendo novamente submetida à análise em uma terceira oficina (Oficina 3 ) que teve como objetivos: atribuir valores aos indicadores e determinar os parâmetros de qualidade a serem considerados na avaliação.

A aferição da pontuação foi feita a partir dos percentuais obtidos. Para o caso de resultados obtidos por escores, foi utilizada a seguinte fórmula para se converter em percentuais:

Percentual final $=\frac{\text { Pontuação obtida }}{\text { Pontuação máxima }} \times 100$

O indicador "proporção de procedimentos odontológicos básicos individuais”, proposto pelo $\mathrm{MS}^{23}$, não foi convertido em percentual para poder ser comparado com resultados obtidos em outras avaliações.

Para a conclusão acerca da qualidade de cada indicador, foi utilizada a seguinte classificação: 0) Crítico; 1) Regular; 2) Aceitável; e 3 ) Satisfatório. Os percentuais que demarcam os parâmetros da classificação para cada indicador foram estabelecidos por consenso entre os participantes das oficinas.

Torna-se importante ressaltar que os indicadores apresentam pontos de cortes diferenciados, sendo que, para os indicadores propostos pelo MS, os valores de referência foram os pactuados pelo município para o ano de 2006.

Para se obter a qualidade final do serviço de odontologia da USF foram somados os escores de todos os indicadores, sendo: Crítico $=0$; Regular $=1$; Aceitável = 2; e Satisfatório $=3$, e aplicada a fórmula acima citada para obtenção do percentual final, o qual obedeceu aos seguintes parâmetros: Crítico de 0 a 24\%; Regular de 25 a $49 \%$; Aceitável de 50 a 74\%; e Satisfatório de 75 a 100\%.

Este estudo foi aprovado pelo Comitê de Ética em Pesquisa (CEP) da UEL.

\section{Resultados}

\section{Modelo-lógico}

O modelo-lógico do serviço de odontologia no município de Cambé pode ser visualizado nas Figuras 1, 2, 3 e 4.

A Unidade Saúde da Família (USF) presta atenção básica partindo do diagnóstico territorial. As famílias da região são cadastradas por Agentes Comunitários de Saúde (ACS), mediante um formulário desenvolvido no próprio serviço, com a finalidade de se coletar informações sobre saúde e condições de vida. Estes cadastros são analisados pelo dentista para a verificação de risco social e biológico. As famílias priorizadas por critérios de eqüidade ${ }^{20}$ recebem visitas domiciliares para ações de promoção de saúde e agendamento na oferta organizada assistencial.

As gestantes do território são convocadas para o atendimento odontológico programado, independentemente do tempo de gravidez, e incluídas no programa assim que iniciam o pré-natal na unidade ou em outra USF. Para as que não comparecem ao pré-natal, é realizada busca ativa, sendo que frequentemente gestantes que fazem prénatal na rede privada procuram o programa. Antes do atendimento clínico, elas são convidadas a participar de uma reunião educativa.

Os indivíduos menores de um ano de idade da área de abrangência da USF são convocados para o atendimento odontológico programado. As vias de acesso à atenção podem ser a visita puerperal, o programa de puericultura da unidade, o programa nacional de imunização, a busca ativa feita pelo ACS, o atendimento programado para gestantes ou ainda por procura espontânea da mãe ao serviço, quando recebem orientações quanto à atenção precoce (importância do atendimento do indivíduo ainda enquanto bebê), aleitamento materno, hábitos e higiene bucal.

Para a faixa etária entre 1 e 14 anos o serviço conta com dois programas: Sorrindo na Creche (SONAC), para 1 a 5 anos, e o Infanto-juvenil para 6 a 14. No Centro de Educação Infantil (CEI) há atendimento clínico preventivo e atividades educativas para o primeiro grupo. Na USF são realizados atendimento clínico e atividades educativas individuais para pessoas de ambos os grupos. Atividades coletivas são realizadas no CEI e nas escolas públicas. 


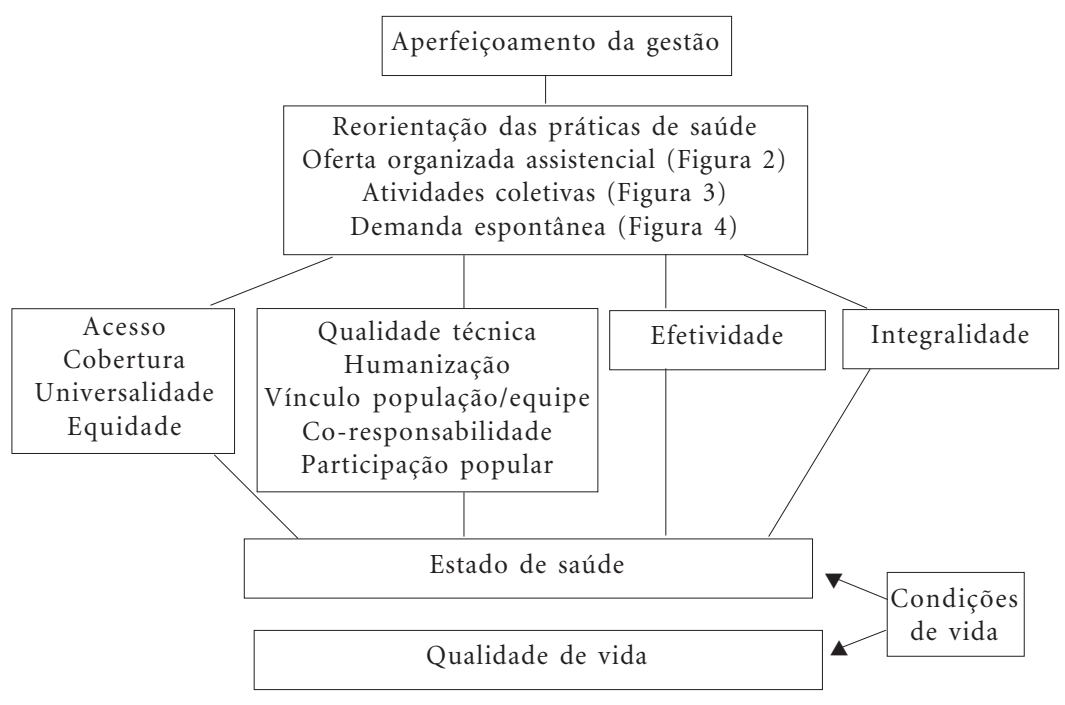

Figura 1. Modelo-lógico da organização do serviço público de odontologia do município de Cambé, PR, 2007.

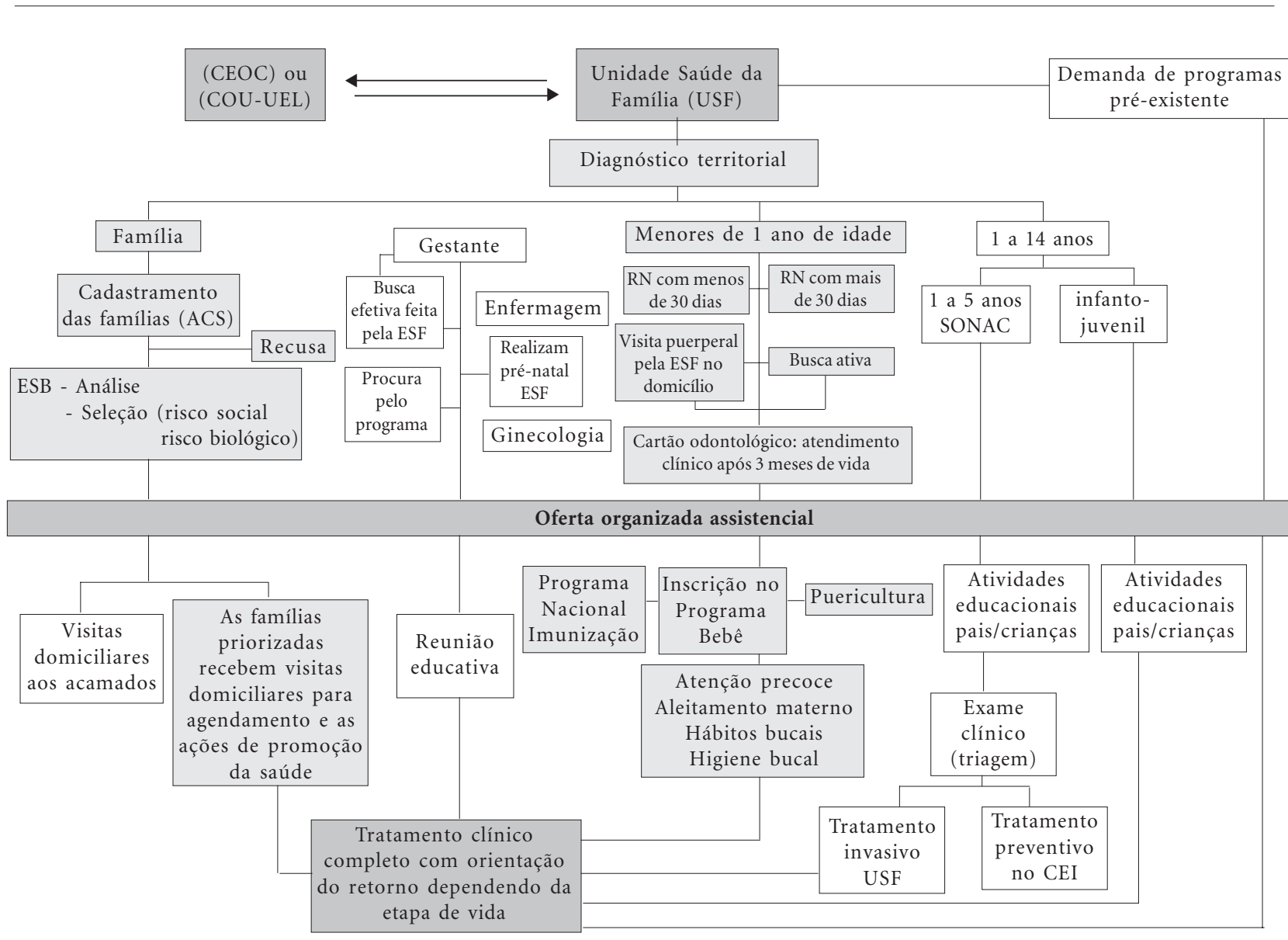

Figura 2. Modelo-lógico da oferta organizada assistencial do serviço público de odontologia do município de Cambé, PR, 2007. 


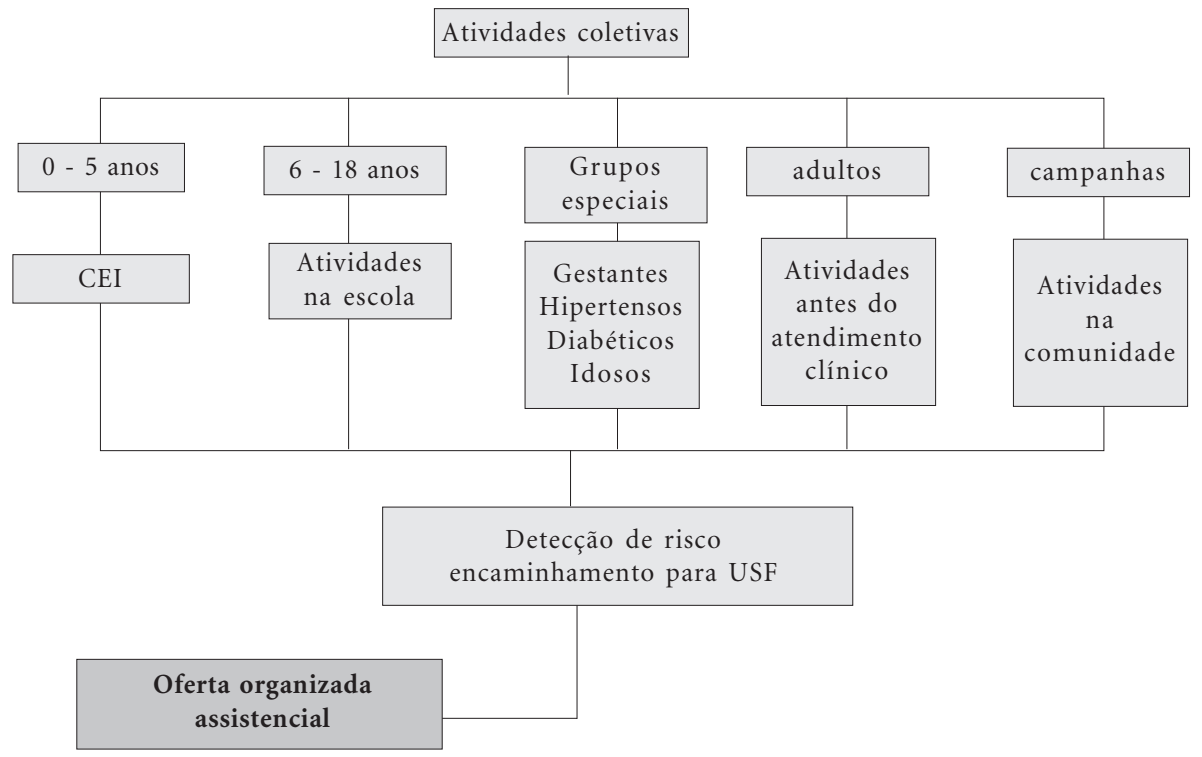

Figura 3. Modelo-lógico das atividades coletivas do serviço público de odontologia do município de Cambé, PR, 2007.

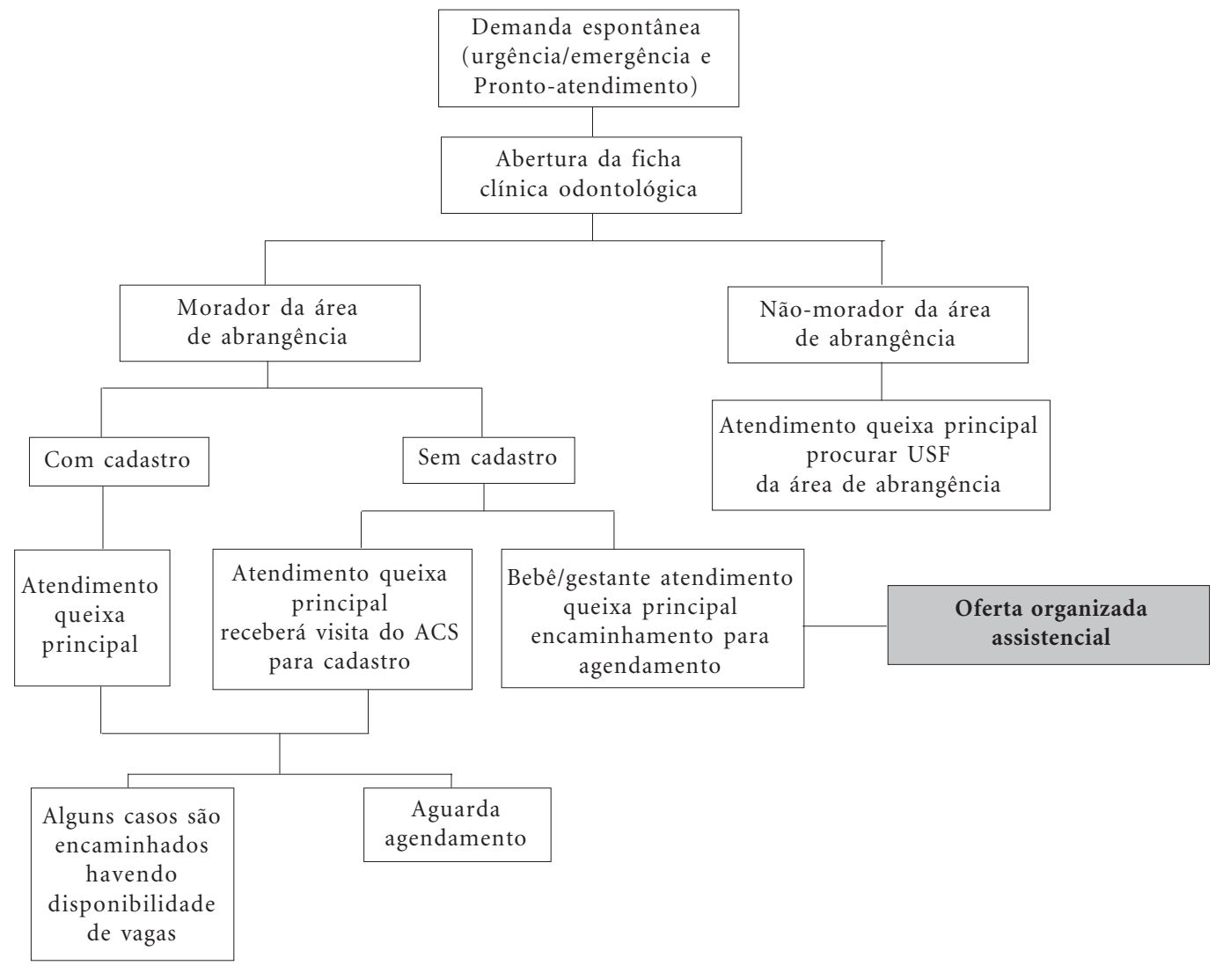

Figura 4. Modelo-lógico do atendimento à demanda espontânea do serviço público de odontologia do município de Cambé, PR, 2007. 
A USF deve manter o atendimento de indivíduos provenientes de programas pré-existentes que ainda não tiveram suas famílias convocadas. Para isto conta com dentistas de apoio que não aderiram ao PSF.

A unidade também deve oferecer atividades coletivas para grupos especiais tais como gestantes, hipertensos, diabéticos e idosos, bem como adultos em geral, aproveitando momentos de campanhas como as de imunização, por exemplo. Nestas ocasiões, se houver detecção de risco pela equipe, o indivíduo deve ser encaminhado para uma USF.

Quanto à demanda espontânea, a unidade oferece vagas tanto para atendimento de emergência/urgência como para pronto-atendimento de procura espontânea. Nestes casos, o indivíduo tem sua queixa principal resolvida e, se ainda não tiver cadastro, é orientado a aguardar cadastramento realizado pelo ACS e posterior agendamento, respeitando a área de abrangência da unidade.

Os casos que exigem tratamento por especialistas são referenciados para o Centro de Especialidades Odontológicas de Cambé (CEOC) e contra-referenciados com registro de informações enviado via malote para a USF de origem.

Todos que acessam a oferta organizada assistencial da odontologia devem ter seu tratamento completo com orientação de retorno dependendo da etapa de vida.

\section{Matriz}

Para a matriz de avaliação do serviço de odontologia do município de Cambé (Quadro 1), foram selecionadas duas dimensões de análise: a primeira avalia o serviço considerando os sujeitos envolvidos no desenvolvimento das práticas de atenção à saúde, e a segunda considera a organização do serviço.

Para a primeira dimensão foram considerados quatro critérios propostos nas oficinas de trabalho: 1) Uso do protocolo; 2) Adequação do quadro técnico às necessidades do serviço; 3 ) Integração da equipe; e 4) Adesão dos agentes às práticas.

Para a segunda dimensão foram considerados oito critérios, também propostos nas oficinas de trabalho: 1) Cobertura de primeira consulta odontológica programática; 2) Proporção de procedimentos odontológicos básicos individuais; 3 ) Cobertura de ação coletiva da escovação dental supervisionada; 4) Diagnóstico territorial; 5) Atendimento domiciliar; 6) Atendimento às gestantes; 7) Atendimento às famílias; 8) Atendimento aos indivíduos.

\section{Discussão}

A institucionalização da avaliação dos serviços de saúde constitui um desafio para todos os níveis de gestão do SUS ${ }^{24,25}$, sendo necessário se aprimorar a prática de monitoramento e avaliação, com o objetivo de contribuir para a tomada de decisões, planejamento, programação e organização do serviço, tendo o cuidado de se levar em conta os sujeitos envolvidos na prática do serviço ${ }^{2,25}$.

No intuito de envolver os profissionais do serviço no desenvolvimento dos indicadores que seriam utilizados na avaliação do município de Cambé, foram propostas oficinas de trabalho. Tais oficinas partiram do pressuposto que o esforço para a construção de consensos em torno do modo de gerir e organizar o processo de trabalho pode ser o ponto de partida para a transformação dos serviços de saúde, ao lado da incorporação de métodos, técnicas e instrumentos que levem em conta a intuição e a criatividade para além das normas e modelos ${ }^{26}$.

No sentido de não limitar o estudo a uma auto-avaliação por parte dos trabalhadores locais, a coleta de informações se estendeu à consulta de documentos oficiais e registros de observações do pesquisador em visitas aos centros de saúde.

Os participantes das oficinas discutiram quais informações poderiam ser utilizadas para aferir a qualidade do serviço e, a partir daí, foram propostos indicadores. Segundo Testa ${ }^{27}$, os indicadores ocupam um lugar intermediário na sequência que vai do dado à informação. Eles aparecem como uma elaboração de dados agrupados e sintetizados de maneira que indiquem elementos importantes da constituição do fenômeno que se estuda. E ainda, para a construção do indicador, realiza-se um processo de abstração, posto que não se tomam todos os elementos do dado registrado, mas somente aqueles que se consideram pertinentes. Para o autor, "o indicador é não somente um sinal, mas também uma base de apoio para a obtenção de informação ou, melhor ainda, de conhecimento" ${ }^{27}$.

Os participantes das oficinas entenderam que a reorientação das práticas depende dos sujeitos para ser implementada e da forma como o serviço deve ser organizado; assim, justificam-se as duas dimensões de análise propostas. 
Quadro 1. Matriz utilizada na avaliação do serviço público de odontologia do município de Cambé, PR, 2007.

\begin{tabular}{|c|c|c|c|c|c|}
\hline Dimensões & Critérios & Indicadores & $\begin{array}{c}\text { Pontuações } \\
\text { (escores ou percentuais) }\end{array}$ & Parâmetros & $\begin{array}{c}\text { Fontes de } \\
\text { informações }\end{array}$ \\
\hline \multirow{4}{*}{ 1. Sujeitos } & 1A. Uso do protocolo & $\begin{array}{l}\text { 1Aa. Conhecimento e } \\
\text { aplicação do protocolo }\end{array}$ & $\begin{array}{l}\text { 0. Não conhece } \\
\text { 1. Conhece e não aplica } \\
\text { 2. Aplica parcialmente } \\
\text { 3. Aplica integralmente }\end{array}$ & $\begin{array}{l}\text { 0. Crítico: } \\
0+25 \% \\
\text { 1. Regular: } \\
25 \%+50 \% \\
\text { 2. Aceitável: } \\
50 \%+75 \% \\
\text { 3. Satisfatório: } \\
75 \% \text { ou mais }\end{array}$ & $\begin{array}{l}\text { 1. Entrevista } \\
\text { com } \\
\text { trabalhadores } \\
\text { da USF } \\
\text { avaliada. } \\
\text { 2. Diário de } \\
\text { campo. }\end{array}$ \\
\hline & $\begin{array}{l}\text { 1B. Adequação do } \\
\text { quadro técnico às } \\
\text { necessidades do } \\
\text { serviço }\end{array}$ & $\begin{array}{l}\text { 1Ba. Houve } \\
\text { esclarecimento para uso } \\
\text { do protocolo }\end{array}$ & $\begin{array}{l}\text { 0. Não } \\
\text { 1. Parcialmente } \\
\text { 2. Sim } \\
\text { 0. Não } \\
\text { 1. Parcialmente } \\
\text { 2. Sim } \\
\text { Percentual obtido }\end{array}$ & $\begin{array}{l}\text { 0. Crítico: } \\
0 \vdash 25 \% \\
\text { 1. Regular: } \\
25 \%+50 \% \\
\text { 2. Aceitável: } \\
50 \%+75 \% \\
\text { 3. Satisfatório: } \\
75 \% \text { ou mais } \\
\text { 0. Crítico: } \\
0 \vdash 25 \% \\
\text { 1. Regular: } \\
25 \%+50 \% \\
\text { 2. Aceitável: } \\
50 \%+75 \% \\
\text { 3. Satisfatório: } \\
75 \% \text { ou mais } \\
\text { 0. Crítico: } \\
0+25 \% \\
\text { 1. Regular: } \\
25 \%+50 \% \\
\text { 2. Aceitável: } \\
50 \%+75 \% \\
\text { 3. Satisfatório: } \\
75 \% \text { ou mais }\end{array}$ & $\begin{array}{l}\text { 1. Entrevista } \\
\text { com } \\
\text { trabalhadores } \\
\text { da USF } \\
\text { avaliada. } \\
\text { 2. Checagem } \\
\text { de registros. } \\
\text { 3. Diário de } \\
\text { campo. }\end{array}$ \\
\hline & $\begin{array}{l}\text { 1C. Integração da } \\
\text { equipe (PSF/ESB) }\end{array}$ & $\begin{array}{l}\text { 1Ca. ESB presente nas } \\
\text { reuniões realizadas na } \\
\text { USF no mês(x reuniões, } \\
\text { presente em y) } \\
\text { 1Cb. Práticas com } \\
\text { equipes } \\
\text { multidisciplinares (x } \\
\text { equipes, presente em y) }\end{array}$ & $\begin{array}{l}\text { Percentual obtido } \\
\text { Percentual obtido }\end{array}$ & $\begin{array}{l}\text { 0. Crítico: } \\
0+25 \% \\
\text { 1. Regular: } \\
25 \%+50 \% \\
\text { 2. Aceitável: } \\
50 \%+75 \% \\
\text { 3. Satisfatório: } \\
75 \% \text { ou mais } \\
\text { 0. Crítico: } \\
0+25 \% \\
\text { 1. Regular: } \\
25 \%+50 \% \\
\text { 2. Aceitável: } \\
50 \%+75 \% \\
\text { 3. Satisfatório: } \\
75 \% \text { ou mais }\end{array}$ & $\begin{array}{l}\text { 1. Entrevista } \\
\text { com } \\
\text { trabalhadores } \\
\text { da USF } \\
\text { avaliada. } \\
\text { 2. Checagem } \\
\text { de registros. } \\
\text { 3. Diário de } \\
\text { campo. }\end{array}$ \\
\hline & $\begin{array}{l}\text { 1D. Adesão dos } \\
\text { agentes às práticas }\end{array}$ & $\begin{array}{l}\text { 1Da. Profissionais se } \\
\text { sentem participantes do } \\
\text { processo de gestão }\end{array}$ & $\begin{array}{l}\text { 0. Não } \\
\text { 1. Parcialmente } \\
\text { 2. Sim } \\
\text { 0. Não } \\
\text { 1. Parcialmente } \\
\text { 2. Sim }\end{array}$ & $\begin{array}{l}\text { 0. Crítico: } \\
0+25 \% \\
\text { 1. Regular: } \\
25 \%+50 \% \\
\text { 2. Aceitável: } \\
50 \%+75 \% \\
\text { 3. Satisfatório: } \\
75 \% \text { ou mais } \\
\text { 0. Crítico: } \\
0+25 \% \\
\text { 1. Regular: } \\
25 \%+50 \% \\
\text { 2. Aceitável: } \\
50 \%+75 \% \\
\text { 3. Satisfatório: } \\
\text { 75\% ou mais }\end{array}$ & $\begin{array}{l}\text { 1. Entrevista } \\
\text { com } \\
\text { trabalhadores } \\
\text { da USF } \\
\text { avaliada. }\end{array}$ \\
\hline
\end{tabular}


Quadro 1. continuação

\begin{tabular}{|c|c|c|c|c|c|}
\hline Dimensões & Critérios & Indicadores & $\begin{array}{c}\text { Pontuações } \\
\text { (escores ou percentuais) }\end{array}$ & Parâmetros & $\begin{array}{c}\text { Fontes de } \\
\text { informações }\end{array}$ \\
\hline \multirow{3}{*}{$\begin{array}{l}\text { 2. Organi- } \\
\text { zação do } \\
\text { serviço } \\
\text { (Indicadores } \\
\text { pactuados } \\
\text { para } \\
\text { avaliação da } \\
\text { atenção } \\
\text { básica) }\end{array}$} & $\begin{array}{l}\text { 2A. Cobertura de } \\
\text { primeira consulta } \\
\text { odontológica } \\
\text { programática. }\end{array}$ & $\begin{array}{l}2 \text { Aa. } \mathrm{n}^{\circ} \text {. total de } \\
\text { primeiras consultas } \\
\text { odontológicas } \\
\text { programáticas realizadas } \\
\text { em determinado local e } \\
\text { período/ população no } \\
\text { mesmo local e período } \\
\text { (x 100) }\end{array}$ & Percentual obtido & $\begin{array}{l}\text { Pactuação }>31 \% \\
\text { 0. Crítico: } \\
0 \vdash 10 \% \\
\text { 1. Regular: } \\
10 \%+20 \% \\
\text { 2. Aceitável: } \\
20 \%+31 \% \\
\text { 3. Satisfatório: } \\
\text { 31\% ou mais }\end{array}$ & $\begin{array}{l}\text { 1. Relatório } \\
\text { de produção } \\
\text { individual. } \\
\text { 2. SIAB. } \\
\text { 3. SIA-SUS }\end{array}$ \\
\hline & $\begin{array}{l}\text { 2B. Proporção de } \\
\text { procedimentos } \\
\text { odontológicos básicos } \\
\text { individuais }\end{array}$ & $\begin{array}{l}2 \mathrm{Ba} \cdot \mathrm{n}^{\circ} \text {. de } \\
\text { procedimentos } \\
\text { odontológicos básicos } \\
\text { individuais realizadas em } \\
\text { determinado local e } \\
\text { período/ população no } \\
\text { mesmo local e período }\end{array}$ & Escore obtido & $\begin{array}{l}\text { Pactuação > } 1,10 \\
\text { 0. Crítico: } \\
0 \vdash 0,36 \% \\
\text { 1. Regular: } \\
0,36 \% \vdash 0,72 \% \\
\text { 2. Aceitável: } \\
0,72 \% \vdash 1,10 \% \\
\text { 3. Satisfatório: } \\
\text { 1,10 ou mais }\end{array}$ & $\begin{array}{l}\text { 1. Relatório } \\
\text { de produção } \\
\text { individual. } \\
\text { 2. SIAB. } \\
\text { 3. SIA-SUS }\end{array}$ \\
\hline & $\begin{array}{l}\text { 2C. Cobertura de } \\
\text { ação coletiva } \\
\text { escovação dental } \\
\text { supervisionada }\end{array}$ & $\begin{array}{l}\text { 2Ca. média anual de } \\
\text { pessoas participantes } \\
\text { na ação coletiva } \\
\text { escovação dental } \\
\text { supervisionada realizada } \\
\text { em determinado local e } \\
\text { período/ população no } \\
\text { mesmo local e período } \\
\text { (x 100) }\end{array}$ & Percentual obtido & $\begin{array}{l}\text { Pactuação > 5\% } \\
\text { 0. Crítico: } \\
0 \vdash 1,6 \% \\
\text { 1. Regular: } \\
1,6 \%+3,2 \% \\
\text { 2. Aceitável: } \\
\text { 3,2\% } \% \text { - 5\% } \\
\text { 3. Satisfatório: } \\
5 \% \text { ou mais }\end{array}$ & $\begin{array}{l}\text { 1. Relatório } \\
\text { de produção } \\
\text { individual. } \\
\text { 2. SIAB. } \\
\text { 3. SIA-SUS }\end{array}$ \\
\hline $\begin{array}{l}\text { 2. Organi- } \\
\text { zação do } \\
\text { serviço } \\
\text { (Indicadores } \\
\text { complemen- } \\
\text { tares) }\end{array}$ & $\begin{array}{l}\text { 2D. Diagnóstico de } \\
\text { demanda (cadastros } \\
\text { feitos pelo ACS) }\end{array}$ & $\begin{array}{l}\text { 2Da. } \mathrm{n}^{\circ} \text {. de cadastros / } \\
\mathrm{n}^{\circ} . \text { de famílias SIAB } \\
(\mathrm{x} \text { 100) } \\
\text { 2Db. no. de cadastros } \\
\text { sem falha de } \\
\text { preenchimento campo } \\
\text { DN / no. de cadastros } \\
\text { não convocados para } \\
\text { tratamento (x 100) } \\
\text { 2Dc. no. de cadastros } \\
\text { analisados pelo CD / } \\
\mathrm{n}^{\circ} \text {. de cadastros (x 100) } \\
\\
\text { 2Dd. no. de cadastros } \\
\text { arquivados de acordo } \\
\text { com protocolo / no. } \\
\text { de cadastros (x 100) }\end{array}$ & Percentual obtido & 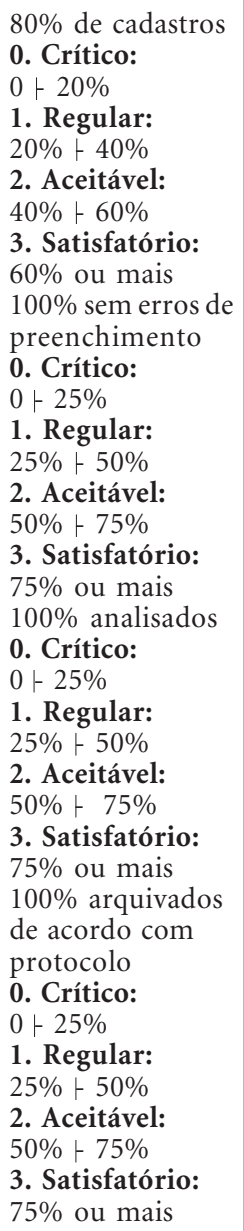 & $\begin{array}{l}\text { 1. Checagem } \\
\text { dos } \\
\text { cadastros. } \\
\text { 2. Entrevista } \\
\text { com } \\
\text { trabalhadores } \\
\text { da USF } \\
\text { avaliada. } \\
\text { 3. SIAB. }\end{array}$ \\
\hline
\end{tabular}


Quadro 1. continuação

\begin{tabular}{|c|c|c|c|c|c|}
\hline Dimensões & Critérios & Indicadores & $\begin{array}{c}\text { Pontuações } \\
\text { (escores ou percentuais) }\end{array}$ & Parâmetros & $\begin{array}{c}\text { Fontes de } \\
\text { informações }\end{array}$ \\
\hline \multirow{4}{*}{$\begin{array}{l}\text { 2. Organi- } \\
\text { zação do } \\
\text { serviço } \\
\text { (Indicadores } \\
\text { comple- } \\
\text { mentares) }\end{array}$} & $\begin{array}{l}\text { 2E. Atendimento } \\
\text { domiciliar(visita } \\
\text { puerperal) }\end{array}$ & $\begin{array}{l}2 \text { Ea. } n^{\circ} \text {. de visitas } \\
\text { puerperais / } n^{\circ} \text {. de } \\
\text { nascidos vivos da região } \\
(\mathrm{x} 100)\end{array}$ & Percentual obtido & $\begin{array}{l}100 \% \text { de visitas } \\
\text { 0. Crítico: } \\
0+25 \% \\
\text { 1. Regular: } \\
25 \%+50 \% \\
\text { 2. Aceitável: } \\
50 \%+75 \% \\
\text { 3. Satisfatório: } \\
\text { 75\% ou mais }\end{array}$ & $\begin{array}{l}\text { 1. Checagem } \\
\text { dos registros. } \\
\text { 2. SINASC. }\end{array}$ \\
\hline & $\begin{array}{l}\text { 2F. Atendimento às } \\
\text { gestantes }\end{array}$ & $\begin{array}{l}2 \mathrm{Fa} . \mathrm{n}^{\circ} \text {. de TC em } \\
\text { gestantes / } \mathrm{n}^{\mathrm{o}} \text {. de } \\
\text { gestantes atendidas pelo } \\
\text { programa (x100) }\end{array}$ & Percentual obtido & $\begin{array}{l}80 \% \text { com } \\
\text { tratamento } \\
\text { concluído (TC) } \\
\text { 0. Crítico: } \\
0 \vdash 20 \% \\
\text { 1. Regular: } \\
\text { 20\% } \vdash 40 \% \\
\text { 2. Aceitável: } \\
40 \% \vdash 60 \% \\
\text { 3. Satisfatório: } \\
\text { 60\% ou mais }\end{array}$ & $\begin{array}{l}\text { 1. Checagem } \\
\text { dos registros. }\end{array}$ \\
\hline & $\begin{array}{l}\text { 2G. Atendimento às } \\
\text { famílias }\end{array}$ & $\begin{array}{l}\text { 2Gb. no. de famílias que } \\
\text { tiveram TC / no. de } \\
\text { famílias SIAB (x 100) } \\
\text { 2Gc. no. de famílias que } \\
\text { tiveram TC / no. de } \\
\text { famílias convocadas (x } \\
\text { 100) }\end{array}$ & $\begin{array}{l}\text { Percentual obtido } \\
\text { Percentual obtido } \\
\text { Percentual obtido }\end{array}$ & 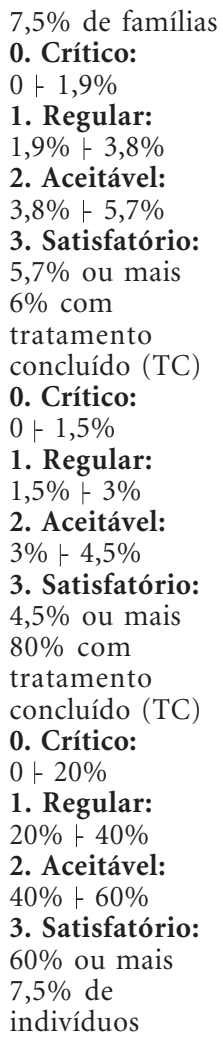 & $\begin{array}{l}\text { 1. Checagem } \\
\text { dos registros. } \\
\text { 2. SIAB. }\end{array}$ \\
\hline & $\begin{array}{l}\text { 2H. Atendimento aos } \\
\text { indivíduos }\end{array}$ & $\begin{array}{l}2 \mathrm{Ha} . \mathrm{n}^{\circ} \text {. de indivíduos } \\
\text { com TC / n }{ }^{\circ} \text {. de } \\
\text { indivíduos SIAB }(\mathrm{x} 100)\end{array}$ & Percentual obtido & $\begin{array}{l}\text { 0. Crítico: } \\
0 \vdash 1,9 \% \\
\text { 1. Regular: } \\
\text { 1,9\% } \vdash 3,8 \% \\
\text { 2. Aceitável: } \\
\text { 3,8\% }>5,7 \% \\
\text { 3. Satisfatório: } \\
\text { 5,7\% ou mais }\end{array}$ & $\begin{array}{l}\text { 1. Checagem } \\
\text { dos registros. } \\
\text { 2. SIAB. }\end{array}$ \\
\hline
\end{tabular}


Quadro 1. continuação

\begin{tabular}{|c|c|c|c|c|c|}
\hline Dimensões & Critérios & Indicadores & $\begin{array}{c}\text { Pontuações } \\
\text { (escores ou percentuais) }\end{array}$ & Parâmetros & $\begin{array}{c}\text { Fontes de } \\
\text { informações }\end{array}$ \\
\hline $\begin{array}{l}\text { 2. Organi- } \\
\text { zação do } \\
\text { serviço } \\
\text { (Indicadores } \\
\text { comple- } \\
\text { mentares) }\end{array}$ & $\begin{array}{l}\text { 2H. Atendimento aos } \\
\text { indivíduos }\end{array}$ & $\begin{array}{l}\text { 2Hb. } \mathrm{n}^{\circ} \cdot \text { de indivíduos } \\
\text { com TC / n } \text {. de } \\
\text { indivíduos convocados } \\
\text { (x100) } \\
\text { 2Hc. no. de indivíduos } \\
\text { menores de } 19 \text { anos } \\
\text { com TC/ no. de } \\
\text { indivíduos menores de } \\
\text { 19 anos no programa } \\
\text { (x100) } \\
\text { 2Hd. no. de indivíduos } \\
\text { maiores de } 19 \text { anos com } \\
\text { TC/ no. de indivíduos } \\
\text { maiores de } 19 \text { anos no } \\
\text { programa (x100) }\end{array}$ & $\begin{array}{l}\text { Percentual obtido } \\
\text { Percentual obtido } \\
\text { Percentual obtido }\end{array}$ & 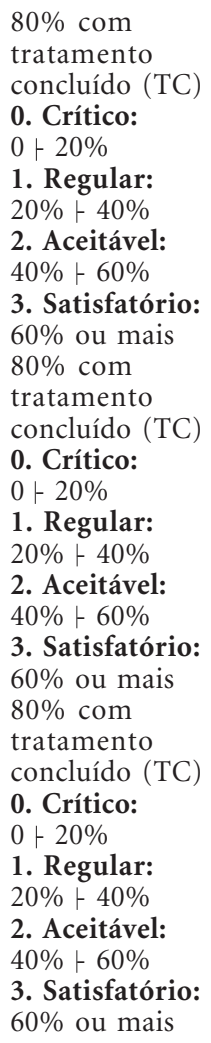 & $\begin{array}{l}\text { 1. Checagem } \\
\text { dos registros. } \\
\text { 2. SIAB. }\end{array}$ \\
\hline
\end{tabular}

Segundo Merhy ${ }^{26}$, no nível dos estabelecimentos e das redes de serviços, no terreno da gestão organizacional e do trabalho, mostra-se que, para superar o modelo médico-hegemônico neoliberal, devem se constituir organizações de saúde, gerenciadas de modo mais coletivo, além de processos de trabalho cada vez mais partilhados, buscando um ordenamento organizacional coerente com uma lógica centrada no usuário, que permita construir vínculos e compromissos estreitos entre os trabalhadores.

$\mathrm{O}$ indicador que se refere ao uso do protocolo parte do princípio de que, à medida que os sujeitos se comprometem em aplicar as rotinas recomendas na organização proposta para o serviço, tendem a conquistar os resultados esperados. Porém, este indicador por si só não poderia responder isto, pois, segundo $\mathrm{Merhy}^{28}$, ao solicitarmos a descrição do serviço a algum trabalhador, a maioria mostra o desenho de um organograma. Este apresenta uma funcionalidade esperada, servindo de parâmetro para as possíveis óticas avaliadoras do cumprimento ou não das funções do "organismo" do serviço; mas outra descrição poderá ser feita, mostrando que o centro de saúde não é um organismo, mas sim uma "arena", onde operam distintos agentes institucionais, com diferentes perspectivas de ação.

Assim sendo, sugerem-se indicadores que procurem mostrar se há uma adequação do quadro técnico às necessidades do sistema por meio de realização periódica de oficinas de Educação Permanente em Saúde ${ }^{29}$, capacitação para executar determinadas tarefas, tais como usar corretamente as rotinas propostas no protocolo ou registrar corretamente informações no sistema. A integração da equipe e a adesão dos agentes às práticas estão refletidas na participação ativa destes sujeitos no processo de gestão e na satisfação em desenvolver suas atividades.

Quanto à dimensão da organização do serviço, o diagnóstico da demanda surge das discussões nas oficinas como a primeira necessidade para se conhecer a comunidade em que se atua e 
estabelecer critérios de acessibilidade, com priorização de problemas visando atingir a equidade no atendimento. Os diagnósticos de demanda são úteis para se definir padrão de morbidade, perfil do usuário dos serviços, entre outros relacionados à assistência ${ }^{30}$. Os cadastros feitos pelos agentes comunitários de saúde devem apresentar qualidade de registro e, posteriormente, serem avaliados e armazenados pelo dentista de forma a contemplar os critérios de eqüidade estabelecidos no protocolo.

$\mathrm{O}$ atendimento às gestantes e a visita puerperal mostraram-se indicadores importantes para o grupo de discussão, uma vez que os indivíduos menores de 12 meses e a gestante são priorizados no atendimento desde a implantação da Clínica do Bebêt ${ }^{15}$.

Quanto aos indicadores propostos pelo MS na portaria n. 493 de 10 de março de $2006^{23}$, foram selecionados: cobertura de primeira consulta odontológica programática, proporção de procedimentos odontológicos básicos individuais e cobertura de ação coletiva escovação dental supervisionada.

Nas oficinas de trabalho foram sugeridas mudanças nos denominadores destes indicadores, uma vez que o Ministério da Saúde utiliza informações do Instituto Brasileiro de Geografia e Estatística (IBGE) e o da avaliação de Cambé optou-se pela utilização de dados do Sistema de Informação da Atenção Básica (SIAB), justificando-se que, para o planejamento de saúde do município, os gestores se orientam pelo SIAB. Segundo dados do gestor e nas discussões nas oficinas, a população cadastrada pelo IBGE está superestimada em relação à cadastrada pelo SIAB. Esta informação foi confirmada em $1^{\circ}$ de abril de 2007, com a divulgação dos resultados da contagem da população do município ${ }^{10}$.

\section{Considerações finais}

A escolha de Cambé como local de estudo favoreceu o desenvolvimento da metodologia determinada, pois o município apresentou uma proposta de organização do serviço de saúde bucal compatível com as Diretrizes da Política Nacional de Saúde Bucal $^{18}$, como se apresenta claramente no Protocolo de Atendimento ${ }^{20}$. Além disso, o consentimento para um estudo de desenvolvimento de indicadores de avaliação, bem como a participação ativa dos trabalhadores locais evidencia um esforço espontâneo por parte do município em prol do aprimoramento do serviço e, em consequência, da atenção aos princípios do SUS.

Por outro lado, a metodologia utilizada proporcionou uma avaliação formativa ao privilegiar a participação dos sujeitos no processo de planejamento da avaliação. Por exemplo, na primeira fase da pesquisa foram identificados problemas no sistema de registro de informações, e este resultado promoveu avanços na discussão da utilização e otimização do protocolo, bem como na questão da informação em saúde.

Finalmente, houve consenso entre os pesquisadores e demais envolvidos no estudo de que a avaliação de um serviço público de odontologia deve ser abrangente e realista para atingir seus objetivos. Para isso, destacou-se a importância da utilização de indicadores desenvolvidos com a participação dos trabalhadores do serviço de odontologia a ser avaliado, além dos indicadores propostos pelo MS e de outros tradicionalmente encontrados na literatura.

Convém ainda mencionar que os indicadores desenvolvidos foram aplicados no serviço e os resultados serão apresentados em outro artigo. 


\section{Colaboradores}

VLRC Bueno participou na elaboração do projeto, na coleta de dados, análise dos resultados e redação do artigo. L Cordoni Júnior oientou a elaboração do projeto, a coleta de dados, a análise dos resultados e participou da redação do artigo. AE Mesas participou da coleta de dados, análise dos resultados e redação do artigo.

\section{Referências}

1. Campos GWS. Há pedras no meio do caminho do SUS! Cien Saude Colet 2007; 12(2):298-299.

2. Arretche MTS. Uma contribuição para fazermos avaliações menos ingênuas. In: Barreira MCRN, Carvalho MCB. Tendências e perspectivas na avaliação de políticas e programas sociais. São Paulo: IEE/ PUC; 2001. p. 43-56.

3. Hartz ZMA. Institutionalizing the evaluation of health programs and policies in France: cuisine internationale over fast food and sur measure over ready-made. Cad Saude Publica 1999; 15(2):229-260.

4. Bodstein R. Atenção Básica na Agenda da Saúde. Cien Saude Colet 2002; 7(3):401-412.

5. Felisberto E. Da teoria à formulação de uma política nacional de avaliação em saúde: reabrindo o debate. Cien Saude Colet 2006; 11(3):553-563.

6. Cericato GO, Garbin D, Fernandes, APS. A inserção da odontologia no PSF: uma revisão crítica das ações e dos critérios de avaliação das Equipes de Saúde Bucal. Rev Fac Odontol 2007; 12(3):18-23.

7. Medina MG, Silva GAP, Aquino R, Hartz ZMA. Uso de modelos teóricos na avaliação em saúde: aspectos conceituais e operacionais. In: Hartz ZMA, Vieira-da-Silva LM, organizadores. Avaliação em saúde: dos modelos teóricos à prática na avaliação de programas e sistemas de saúde. Rio de Janeiro: Editora Fiocruz; 2005. p. 41-63.

8. Yin RK. Estudo de caso: planejamento e métodos. Porto Alegre: Editora Bookman; 2001.

9. Denis J, Champagne F. Análise da implantação de programas. In: Hartz ZMA, organizador. Avaliação em saúde: dos modelos conceituais à prática na análise da implantação de programas. Rio de Janeiro: Editora Fiocruz; 1997. p. 49-88.

10. Instituto Brasileiro de Geografia e Estatística (IBGE). Contagem da população. [acessado 2007 nov 27]. 2007. Disponível em: http://www.ibge.gov.br/home/ estatistica/populacao/contagem2007/PR.pdf

11. Prefeitura Municipal de Cambé. Dados econômicos e sociais. Cambé; 2008. [acessado 2008 mar 31]. Disponível em: http://www.cambe.pr.gov.br

12. Brasil. Ministério da Saúde. Secretaria de Vigilância em Saúde. Departamento de Análise de Situação de Saúde. Vigilância em Saúde: dados e indicadores selecionados (ano 4, n. 4, nov. 2006). Brasília: Ministério da Saúde; 2003.

13. Paraná. Secretaria de Estado de Educação. Programa Paraná Alfabetizado. [acessado 2008 mar 31]. Disponível em: http://www.paranaalfabetizado.pr. gov.br

14. Nakagawa MCS. Saúde bucal no PSF: estratégia para a universalização e a integralidade no município de Cambé [monografia]. Cambé (PR): Secretaria Municipal da Saúde; 2006.

15. Issao M, Ferelle A, Walter LRF. Odontologia para o bebê: odontopediatria do nascimento aos 3 anos. São Paulo: Editora Artes Médicas; 1996. 
16. Londrina. Prefeitura Municipal. Levantamento epidemiológico de cárie nas idades de 5 e 12 anos e fluorose dentária na idade de 12 anos, em escolares da rede pública e particular de ensino, zona urbana e rural, em Londrina, Cambé e Ibiporã - Paraná. [acessado 2007 ago 14]. Disponível em: http://www. saude.pr. gov.br/saudebucal

17. Brasil. Ministério da Saúde. Portaria n. 1.444. Estabelece incentivo financeiro para reorganização da saúde bucal prestada nos municípios por meio do Programa Saúde da Família. Diário Oficial da União 2000; $29 \mathrm{dez}$.

18. Brasil. Ministério da Saúde. Secretaria de Atenção à Saúde. Departamento de Atenção Básica. Coordenação de Saúde Bucal. Diretrizes da Politica Nacional de Saúde Bucal. Brasília: Ministério da Saúde; 2004.

19. Conselho Federal de Odontologia. Prêmio Brasil Sorridente vai para Araguaína, Tocantins. J Consetho Fed Odontol [periódico na Internet]; 2006; [acessado 2006 ago 27]; 14(70). Disponível em: http:// www.cfo.org.br/jornal/n70/default.asp

20. Prefeitura Municipal de Cambé. Secretaria Municipal de Saúde. Departamento de Atenção Integral à Saúde. Divisão de Serviços Odontológicos. Protocolo de atenção odontológica no município de Cambé - PR. Cambé; 2006.

21. Vieira-da-Silva LM, Hartz ZMA, Chaves SC, Silva GAP. Metodologia para análise da implantação de processos relacionados à descentralização da atenção à saúde no Brasil. In: Hartz ZMA, Vieira-daSilva LM, organizadores. Avaliação em saúde: dos modelos teóricos à prática na avaliação de programas e sistemas de saúde. Rio de Janeiro: Editora Fiocruz; 2005. p. 207-253.

22. Anastasiou LGC, Alves LP, organizadores. Processos de ensinagem na universidade: pressupostos para as estratégias de trabalho em aula. Joinville: Editora Univille; 2007.

23. Brasil. Ministério da saúde. Portaria n. 493/GM Aprova a relação de Indicadores da Atenção Básica - 2006, cujos indicadores deverão ser pactuados junto aos municípios, estado e Ministério da Saúde. Diário Oficial da União 2006; 11 mar.
24. Novaes, HMD. Avaliação de programas, serviços e tecnologias em saúde. Rev. Saude Publica 2000; 34(5):547-549.

25. Teixeira CF. Institucionalizando a prática de avaliação em saúde: significado e limites. Cien Saude Colet 2006; 11(3):572-574.

26. Merhy EE. Saúde: a cartografia do trabalho vivo. São Paulo: Editora Hucitec; 2005.

27. Testa M. Pensamento estratégico e lógica de programação: o caso da saúde. São Paulo: Editora Hucitec; 1995.

28. Merhy EE. Em busca de ferramentas analisadoras das tecnologias em saúde: a informação e o dia-adia de um serviço, interrogando e gerindo o trabalho em saúde. In: Merhy EE, Onocko R, organizadores. Agir em saúde: um desafio para o público. São Paulo: Editora Hucitec; 2002. p. 113-150.

29. Brasil. Ministério da Saúde. Portaria n. 198/GM/ MS. Institui a política nacional de Educação Permanente em Saúde como estratégia do Sistema Único de Saúde para formação e do desenvolvimento de trabalhadores para o setor e dá outras providências. Diário Oficial da União 2004; 14 fev.

30. Takeda S. A organização de serviços de atenção primária à saúde. In: Duncan BB, Schmidt MI, Giugliani E, editores. Medicina ambulatorial: condutas de atenção primária baseadas em evidências. Porto Alegre: Artmed; 2004. p. 22-29.

Artigo apresentado em 07/05/2009

Aprovado em 08/10/2009

Versão final apresentada em 01/11/2009 